\title{
Stretchable and Waterproof Self-Charging Power System for Harvesting Energy from Diverse Deformation and Powering Wearable Electronics
}

Fang $\mathrm{Yi}^{\perp_{1,2}}$, Jie Wang ${ }^{\perp_{1,3}}$, Xiaofeng Wang ${ }^{\perp_{1,4}}$, Simiao Niu ${ }^{l}$, Shengming $\mathrm{Li}^{l}$, Qingliang Liao ${ }^{2}$, Youlong Xu ${ }^{3}$, Zheng You ${ }^{4}$, Yue Zhang ${ }^{2}$, and Zhong Lin Wang ${ }^{1,5}$

1

School of Materials Science and Engineering, Georgia Institute of Technology, Atlanta, Georgia 30332-0245, United States

E-mail: zlwang@gatech.edu

${ }^{2}$ State Key Laboratory for Advanced Metals and Materials, School of Materials Science and Engineering, Beijing Municipal Key Laboratory of New Energy Materials and Technologies, University of Science and Technology Beijing, Beijing 100083, China

E-mail: yuezhang@ustb.edu.cn

${ }^{3}$ Electronic Materials Research Laboratory, Key laboratory of the Ministry of Education and International Center of Dielectric Research, Xi'an Jiaotong University, Xi'an 710049, China

${ }^{4}$ Department of Precision Instrument, Tsinghua University, Beijing 100084, China

${ }^{5}$ Beijing Institute of Nanoenergy and Nanosystems, Chinese Academy of Sciences, Beijing, 100083, China 
Note S1 Effect of TENG's electrode resistance on its performance.

When the electrode resistance of TENG cannot be neglected, the power applied to the external resistance can be calculated as: (For simplification, the open-circuit voltage $\left(\mathrm{V}_{\mathrm{oc}}\right)$ is assumed as a sinusoidal profile in the analysis.)

$$
P=\frac{V_{o c}{ }^{2} R}{\frac{1}{\omega^{2} C^{2}}+(r+R)^{2}}
$$

where $\mathrm{R}$ is the external resistance, $\mathrm{r}$ is TENG's electrode resistance, $\mathrm{C}$ is TENG's inherent capacitance. It can be seen that when $r$ is smaller than $1 / 100 \omega \mathrm{C}$, the impact of $\mathrm{r}$ on $\mathrm{P}$ is very small. Generally, TENG's inherent impedance is in the range of hundreds of $M \Omega$, therefore the performance of TENG will change very slightly as long as TENG's electrode resistance is smaller than the $\mathrm{M} \Omega$ range.

Note S2 Detailed calculation process for the equivalent galvanostatic current.

The equivalent galvanostatic current can be calculated as:

$$
I=C \times V / t
$$

where $\mathrm{C}$ is the charging capacitance, $\mathrm{t}$ is the charging time and $\mathrm{V}$ represents the voltage window.

For the self-charging power system, the $\mathrm{C}$ of the two SCs in series is around $194 \mu \mathrm{F}$.

Thus, the equivalent galvanostatic current for Figure $4 \mathrm{a}$ can be acquired: $\mathrm{I} \approx 337 \mathrm{nA}$. 
Figure S1 (a) Dependence of triboelectric nanogenerator's electrode resistance on the tensile strain. (b) Dependence of supercapacitor's electrode resistance on the tensile strain.
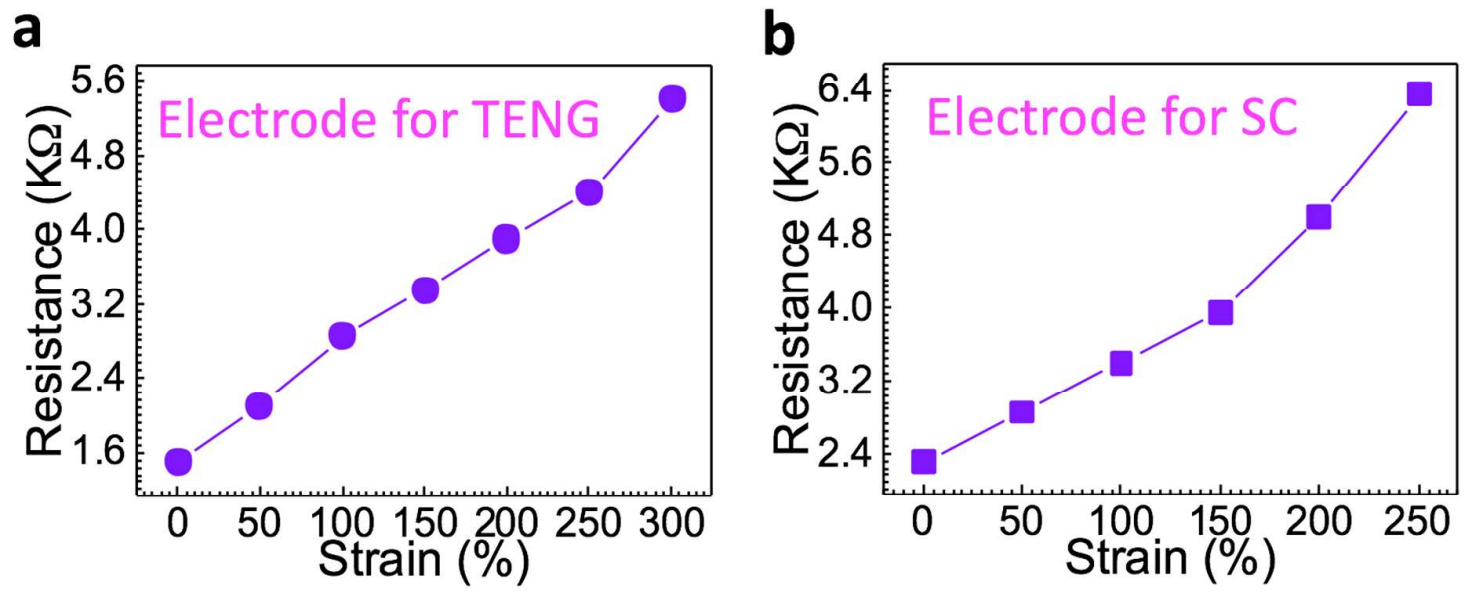
Figure S2 Schematic deptiction of the fabrication process of the self-charging power system.
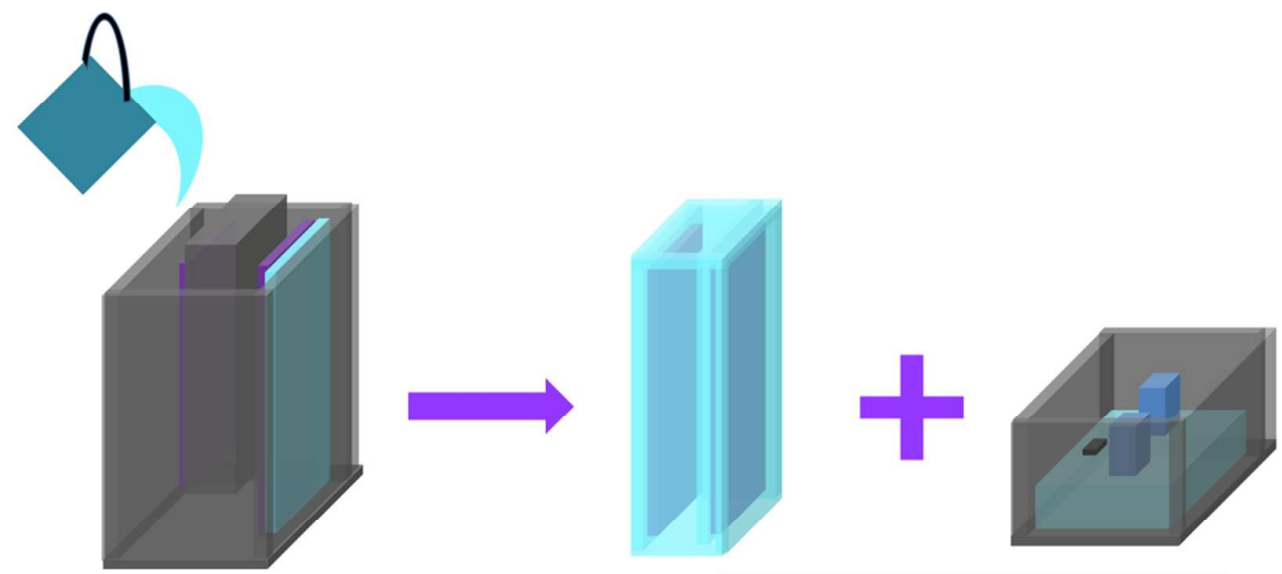

Mold

Silicone Rubber

- Electrode

- Supper capacitor

\section{Rectifier}
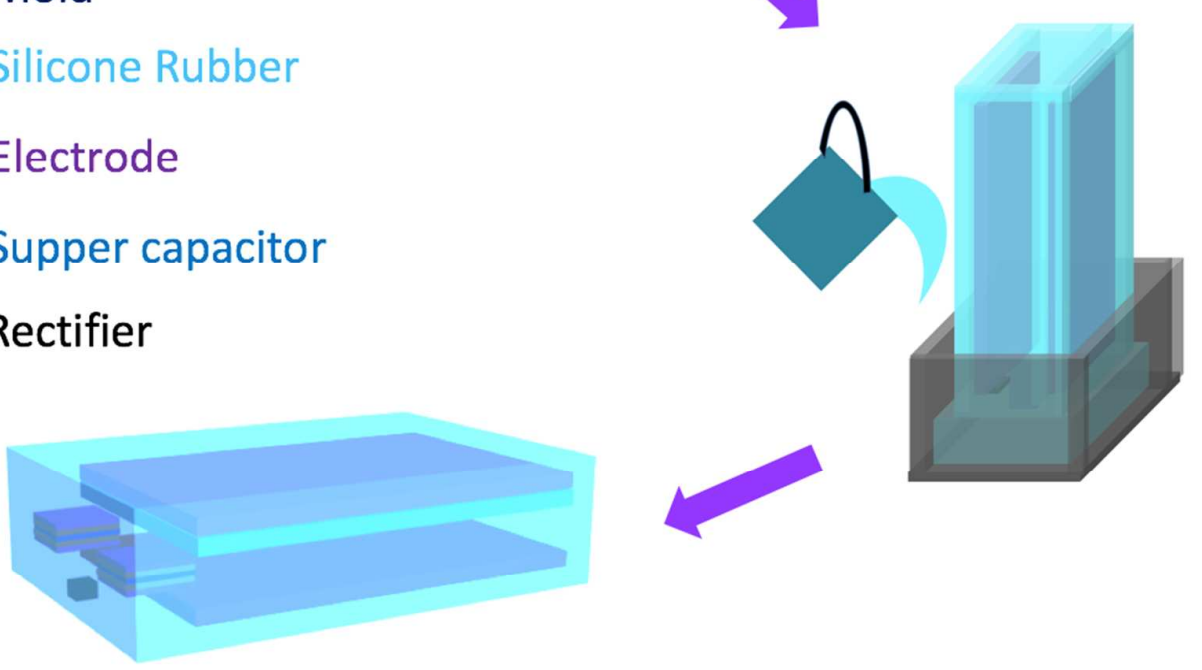
Figure S3 The measured open-circuit voltage $\left(\mathrm{V}_{\mathrm{oc}}\right)$ and the short-circuit current density $\left(\mathrm{J}_{\mathrm{sc}}\right)$ upon (a) pressing, (b) stretching, (c) bending, and (d) twisting motion.

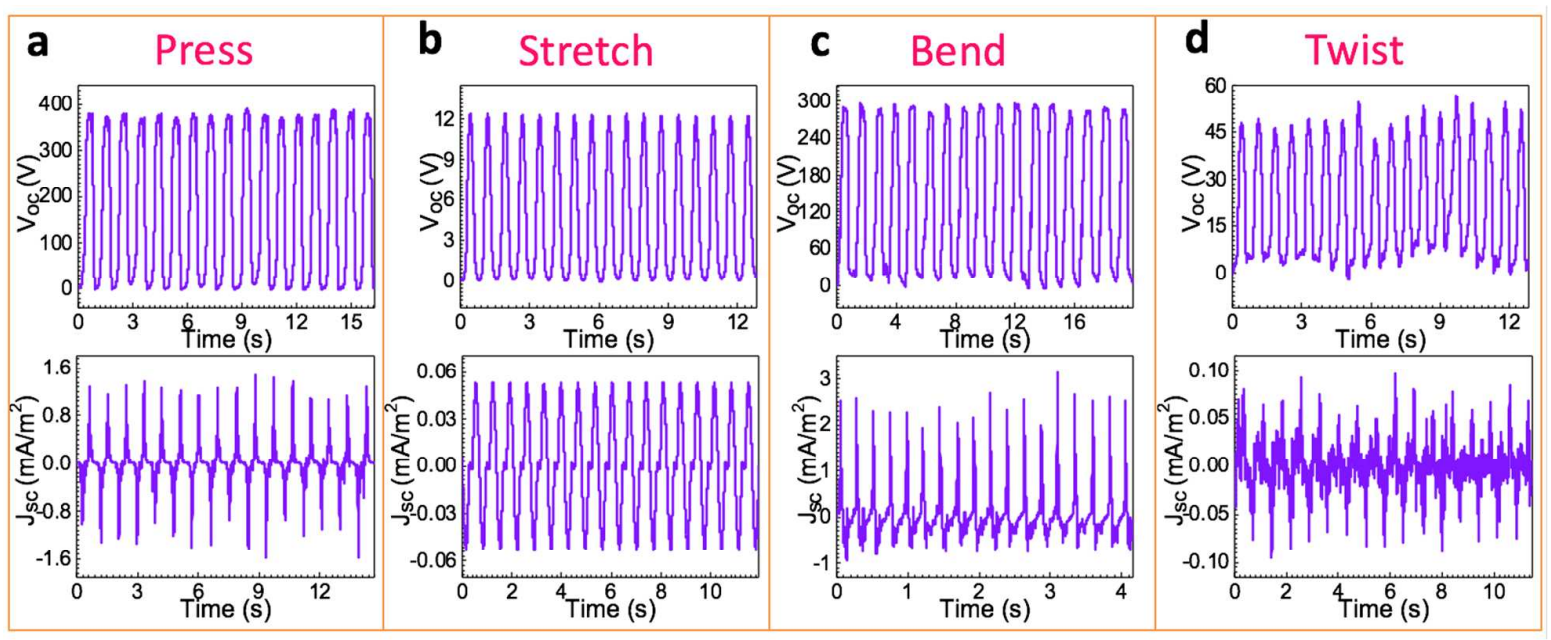


Figure S4 Schematic diagrams showing the working mechanism of the triboelectric nanogenerator part when it is under (a) pressing, (b) stretching, (c) bending, and (d) twisting motion.

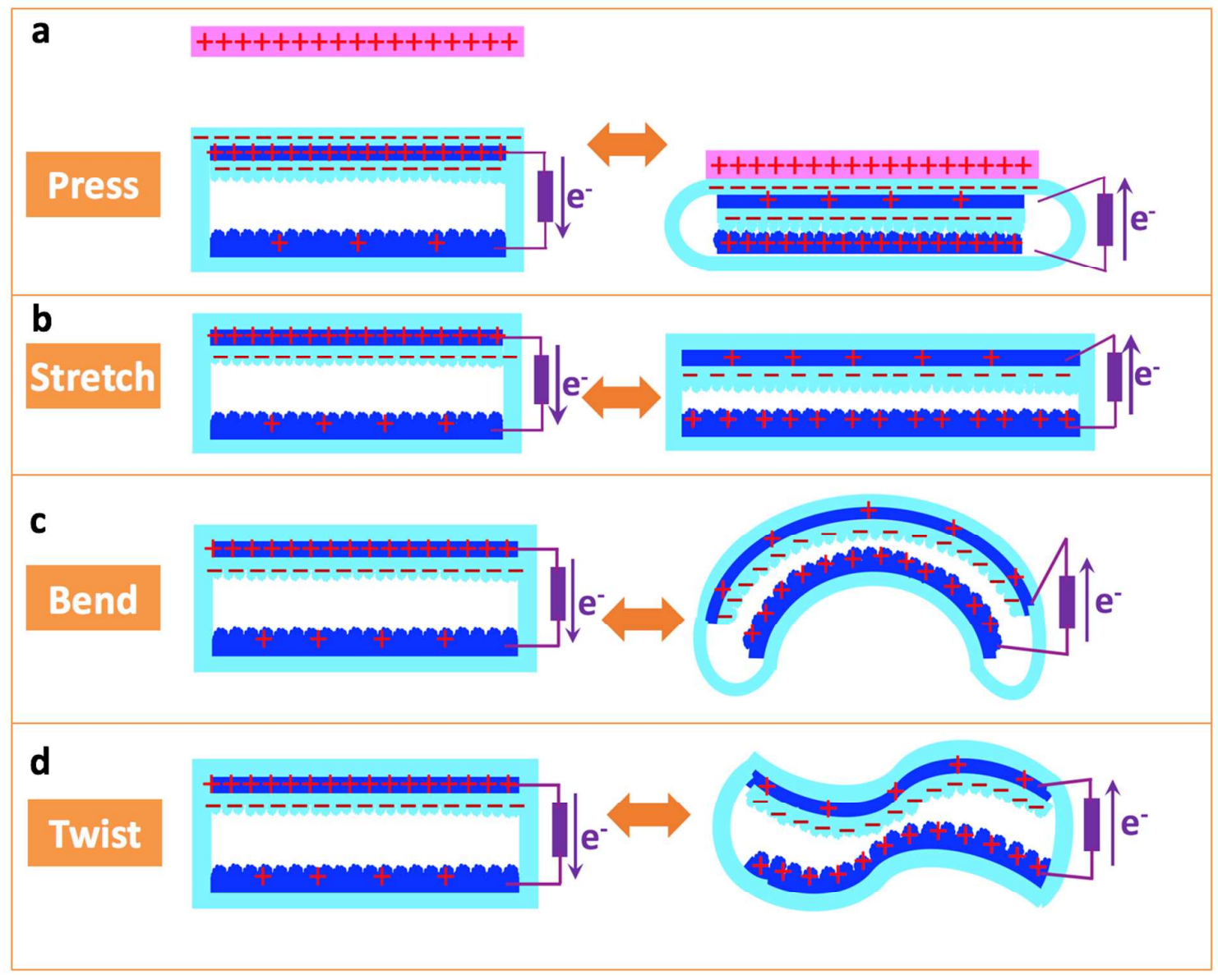


Figure S5 (a) Dependence of the open-circuit voltage $\left(\mathrm{V}_{\mathrm{oc}}\right)$ on the distance between the contacting object and the device. (b) The summarized relationship between the $\mathrm{V}_{\mathrm{oc}}$ and the elongation of the device.
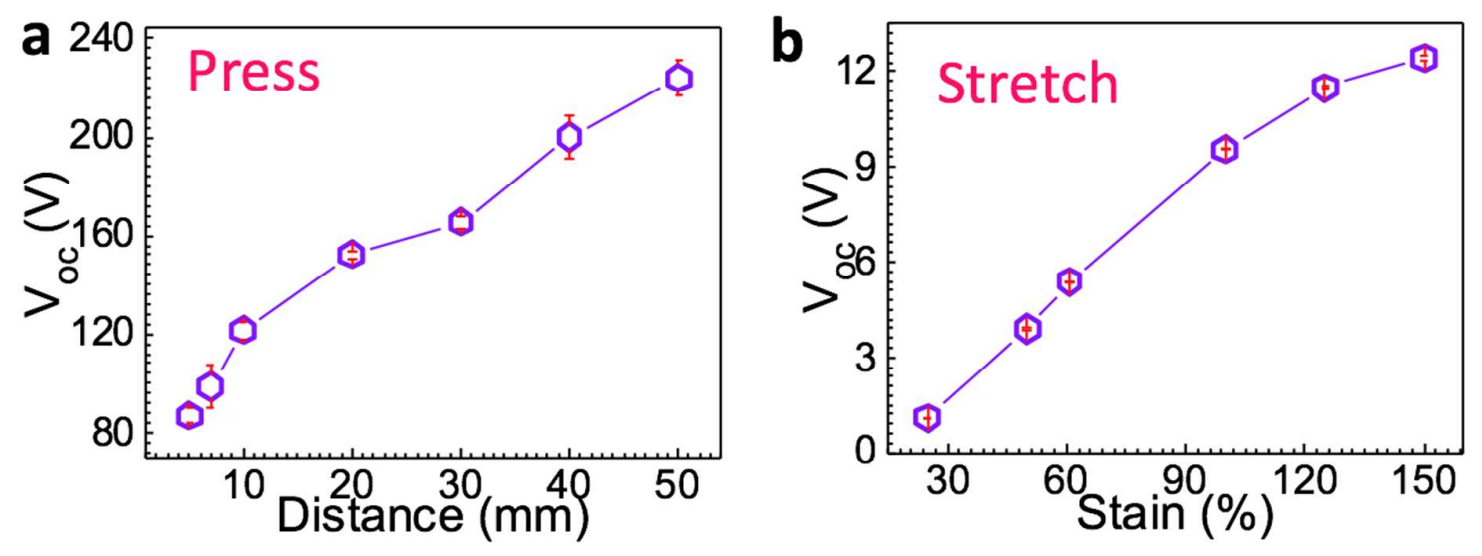


\section{Supporting Movies}

Supporting Movie S1 Powering LEDs with the triboelectric nanogenerator part by patting the shoulder.

Supporting Movie S2 Powering LEDs with the triboelectric nanogenerator part by bending the arm.

Supporting Movie S3 Powering LEDs with the triboelectric nanogenerator part by rotating the wrist.

Supporting Movie S4 The waterproof property of the stretchable self-charging power system.

Supporting Movie S5 Powering a wearable electronic watch by the stretchable self-charging power system. 